\title{
La posmodernidad en los fundamentos del sistema educativo costarricense
}

\section{Postmodernism in the foundations of the Costa Rican educational system}

Yors Guillermo Solís Vargas

Ministerio de Educación Pública, Costa Rica

yors.solis.vargas@mep.go.cr

Recibido: 29/06/2020 • Aceptado:20/05/2021

\begin{abstract}
Resumen
El siguiente ensayo tiene como propósito principal argumentar a favor de que el paradigma de la complejidad, nuevo fundamento de la actual Política Educativa de Costa Rica, es posmodernista. Para este propósito, se utilizó una metodología de revisión de textos, entre los cuales, están documentos filosóficos, políticos, educativos y artículos académicos. Al final, después de exponer una serie de reflexiones críticas, se cerró el texto con una breve conclusión, visualizada desde la posmodernidad y el pensamiento complejo, que invita a reflexionar sobre la educación de dicho país.
\end{abstract}

Palabras clave: Posmodernidad, educación, Costa Rica, paradigma de la complejidad.

\section{Abstract}

This essay is intended to establish that the paradigm of complexity, which is an element in the new foundation of current educational policy in Costa Rica, is postmodernist. To do so, a methodology of textual analysis is used. The texts include philosophical, political, and educational documents 
and academic articles. After presenting a series of critical reflections, the essay concludes with an invitation to reflect on education in this country as it is visualized from the points of view of postmodernism and complex thinking.

Keywords: Postmodernism, education, Costa Rica, paradigm of complexity.

\section{Introducción}

En la actual Política Educativa de Costa Rica, publicada por el Ministerio de Educación Pública (MEP) (2017a), se establece el paradigma de la complejidad como nuevo fundamento filosófico y conceptual de la educación nacional. En este documento no se deja claro qué significa, cómo se traduce exactamente a nuestro contexto o por qué se eligió este paradigma. Ni allí, ni en los distintos programas de enseñanza se puede dilucidar de dónde procede ni a quién se le atribuye este modelo.

Aunque haya autores fuera de Costa Rica que han considerado que este paradigma no es posmodernista (Caro, 2002; Munné, 2001; Solana, 2008), en este ensayo se va a argumentar a favor de que este modelo sí lo es y, con la adopción de este como fundamento del sistema educativo de Costa Rica, la enseñanza en este país se enrumba, más de lo que ya está hacia una visión esencialmente posmodernista del mundo.

Era de esperarse que esta nueva visión de las ciencias llegara a las bases de la educación costarricense, ya que desde finales del siglo XX la Organización de las Naciones Unidas para la Educación, la Ciencia y la Cultura (Unesco) ha extendido las ideas del pensamiento complejo en la educación latinoamericana.

En vista de que Costa Rica ha firmado convenios, tratados y agendas internacionales; depende, en muchos casos, de lo que estas organizaciones, instituciones, bancos, agencias, etc., extranjeros le exija. Debe aceptar, adaptar y poner en práctica los modelos (no solo educativos) que estos convenios internacionales planteen a pesar de que el país no esté preparado para lo que se exige.

En la educación de Costa Rica esto ha sido siempre común y, con la actual Política Educativa (MEP, 2017a), no es la excepción, ya que al incluir 
el pase al producto intelectual del "primer mundo" que, probablemente, por la exigencia de políticas internacionales o por la simple admiración, tienen siempre ganada la última palabra, lo cual reafirma, penosamente, el subdesarrollo y la neocolonización de la que es parte Costa Rica ante el hecho de no generar, ni promover la generación, de sus propias formas de enseñanza.

Al dejar de lado las distintas valoraciones que ha tenido la posmodernidad en diversos campos del conocimiento, hace falta reflexionar críticamente debido a que oficialmente hay en la Política Educativa costarricense un paradigma como este, el cual, si es lo más conveniente, que se utilice como fundamento un modelo así (extranjero y posmodernista) o si se debe repensar la inclusión en la educación de una visión fundamentalmente posmoderna.

Al margen de si este cambio paradigmático es el más adecuado, es el correcto o es el idóneo para el contexto costarricense, este ensayo va a invitar a pensar, a dudar, a reflexionar críticamente y, si se quiere, a tomar partida o no, de este nuevo modelo que se presenta en la educación oficial de Costa Rica.

Siempre que se está ante un paradigma nuevo en las ciencias, da la impresión de que el pensamiento no encuentra un mejor modelo para concebir las distintas disciplinas científicas. Parece ser que, con el fluir de los tiempos, la necesidad, forzada o no de un cambio permite iniciar una nueva manera de pensar que se muestra más apta que anteriores formas de examinar la vida. Una vez más, Costa Rica se inclina por los estudios, teorías o paradigmas educativos extranjeros — principalmente europeos o del "primer mundo" 1- que se erigen como novedosos e idóneos para todo el planeta, ante los cuales Latinoamérica siempre acoge con veneración y docilidad.

En las dos últimas décadas, en este país se ha investigado poco sobre el paradigma de la complejidad. Según Gallegos (2016), hasta el año 2016 la Universidad de Costa Rica ha publicado seis artículos; mientras que la Universidad Nacional, dos en relación con la complejidad moriniana. Sin embargo, aunque no es propio ni se ha investigado tanto como se pensaría para incluirlo como parte de una política educativa, en 2017 se

1 Como por ejemplo el constructivismo de Piaget, el socioconstructivismo de Vigotsky, el aprendizaje significativo de Ausubel, la teoría de las inteligencias múltiples de Gardner, los estudios inter y transdisciplinarias aplicados a la educación (ciencias cognitivas o neuroeducación). 
oficializó en el sistema educativo nacional como fundamento de la educación costarricense.

\section{El paradigma de la complejidad}

El paradigma de la complejidad se funda en el concepto de pensamiento complejo propuesto por Edgar Morín en algunas de sus múltiples publicaciones. La publicación que mejor sintetiza este paradigma es Introducción al pensamiento complejo (Morin, 1994), el cual representa el texto base para este apartado.

Este paradigma hace referencia a la idea de que la realidad es compleja, multifacética, multidimensional, multirreferente, etc., y no puede ser, como creía la ciencia moderna, reducida o simplificada, ya que, para Morin (1994), esta es un tejido de constituyentes heterogéneos que conforman el mundo, el cual es complejo y sus partes no se pueden separar. Según este concepto, la realidad es como una tela que está entrelazada por miles de fibras que no se pueden destejer para analizarse y comprenderse mejor.

La complejidad se presenta con los rasgos inquietantes de lo enredado, de lo inextricable, del desorden, la ambigüedad, la incertidumbre... De allí la necesidad, para el conocimiento, de poner orden en los fenómenos rechazando el desorden, de descartar lo incierto, es decir, de seleccionar los elementos de orden y de certidumbre, de quitar ambigüedad, clarificar, distinguir, jerarquizar... Pero tales operaciones, necesarias para la inteligibilidad, corren el riesgo de producir ceguera si eliminan a los otros caracteres de lo complejo (Morin, 1994, p. 17).

Para Morin (1994), la ciencia debe reconocer la incompletitud, lo incierto y lo inacabado, así como la contradicción y la incertidumbre del conocimiento humano frente a la complejidad, para que a través de un proceso dialéctico aspire a un conocimiento multidimensional, multirreferencial en lugar de un uno unidimensional. De esta manera, lo que se pretende es defender la idea de que la transdisciplinariedad en las ciencias, a través de procesos dialógicos con diversos discursos de distintas especialidades científicas, es necesaria para que el pensamiento no se centre en un conocimiento especial, parcializado, que mutila la realidad. 
En la ciencia moderna ha reinado la simplificación y reducción del conocimiento, esto, según Morin (1994), ha contraído una patología:

La patología de la razón es la racionalización, que encierra a lo real en un sistema de ideas coherente, pero parcial y unilateral, y que no sabe que una parte de lo real es irracionalizable, ni que la racionalidad tiene por misión dialogar con lo irracionalizable. [...] La ciencia construye su objeto extrayéndolo de su ambiente complejo para ponerlo en situaciones experimentales no complejas (pp. 34-35).

Es por esto que Morin (1994), expone una nueva conceptualización de la realidad como realidad compleja. Para ello,retoma planteamientos como el de la Teoría de Sistemas, la Cibernética, el Teorema de la incompletitud de Gödel, la física cuántica, el sistema abierto y el segundo principio de la Termodinámica (planteamientos que alteran el determinismo científico moderno) para luego esbozar su método que se entiende como "un proceso en curso de búsqueda de estrategias viables para un pensar complejo físico-bioantropológico desde una perspectiva científico-filosófico-literaria, que permita una praxis ética en el campo tanto del conocimiento académico como de la praxis social" (Morin,1994, p. 8).

La ciencia, desde René Descartes, ha dividido la realidad en dos partes: sujeto y objeto, además, ha privilegiado al objeto por encima del sujeto. Sin embargo, de acuerdo con Morin (1994), el sujeto y el objeto aparecen como inseparables en relación con el sistema que representa la realidad. Para Morin (1994), la complejidad escapa de la "disyunción y de la anulación del sujeto y del objeto porque hemos partido del concepto de sistema abierto, que implica en sí mismo, ya en su carácter más elemental, la presencia consustancial del ambiente, es decir, la interdependencia sistema eco-sistema" (p. 41).

La scienza nuova, como la llama Morin (1994), intenta unificar la ciencia bajo el concepto de auto-eco-organización en el que la física, la biología y la antropología dejan de ser disciplinas cerradas, aunque sin perder su identidad, pero al mismo tiempo, sin caer en doctrinas dogmáticas como el fisicismo, el biologismo, el antropologismo. "Lo que queremos desarrollar ahora, más allá del reduccionismo y del holismo, es la idea de unidad 
compleja, que enlaza al pensamiento analítico-reduccionista y al pensamiento global, en una dialectización" (Morin, 1994, p. 81).

El pensamiento complejo de Morin no pretende dar respuestas o recetas para hacer ciencia (como lo planteaba el método científico de la ciencia moderna), lo que propone este autor es el desafío de pensar complejamente como metodología para los distintos quehaceres de la vida. Al pensar de esta manera: la indeterminación, la emergencia, las interferencias, el azar, lo científico, lo no científico, así como las distintas teorías de las distintas disciplinas científicas, son ahora importantes para generar un diálogo sobre las diversas dimensiones de la realidad, logrando con esto la unidad de la multiplicidad, la unitas multiplex (Morin, 1994).

\section{El posmodernismo y la posmodernidad}

El término posmodernismo usualmente se ha utilizado para describir algunos movimientos artísticos y culturales ocurridos el siglo anterior, sin embargo, su concepto también se ha asociado en general a un despreocupado pluralismo de estilos y a la crítica contra la modernidad. En la Enciclopedia Oxford de filosofía el postmodernismo (o posmodernismo) se define como:

[U]na variedad de contextos (arquitectura, música, poesía, ficción, etc.) para cosas que parecen estar relacionadas todo lo más por un despreocupado pluralismo de estilos y por un vago deseo de haber realizado algo con pretensiones de alta cultura modernista. En términos filosóficos, el posmodernismo tiene algo en común con la crítica de los valores de la llustración y de las pretensiones de verdad" (Norris, 2008, p. 932).

Aunque Habermas (1985), afirma que antes de negar, atacar o abandonar la modernidad se debería de aprender de los equívocos e intentar completar el proyecto que presentó. Por otro lado, Díaz (2000), afirma que se está en una nueva época histórica: la posmodernidad (capitalismo tardío, época posindustrial, edad digital, etc., son otros nombres con los que también se le conoce a este nuevo periodo, según esta autora), la cual agotó su anterior época, la modernidad, durante el desarrollo del siglo XX: 
El discurso de la modernidad se refiere a leyes universales que constituyen y explican la realidad. Algunos de sus términos son determinismo, racionalidad, universalidad, verdad, progreso, emancipación, unidad, continuidad, ahorro, mañana mejor. El discurso de la posmodernidad, en cambio, sostiene que sólo puede haber consensos locales o parciales (universales acotados), diversos juegos de lenguaje o paradigmas inconmensurables entre sí. Algunos de sus términos son deconstrucción, alternativas, perspectivas, indeterminación, irreversibilidad, descentralización, disolución, diferencia. La modernidad fue rica en "ismos": iluminismo, modernismo, empirismo, racionalismo, positivismo; la posmodernidad es rica en "pos": posestructuralismo, posindustrial, poscrítica, poshistoria, posciencia, posfilosofía, postsexualidad (Díaz, 2000, pp. 15-16).

Para el tema que aquí interesa, el paradigma de la complejidad como fundamento posmodernista en la educación costarricense, y siguiendo a Díaz (2000) con su conceptualización de la posmodernidad, se puede decir que, en vista de que el paradigma de la complejidad riñe con el determinismo, la racionalidad, la universalidad, la dualidad sujeto-objeto, la verdad única; plantea consensos parciales del conocimiento, relativiza el concepto de verdad, toma la incertidumbre, lo indeterminado o el azar como elementos importantes para el conocimiento científico, al promover la multidimensionalidad y la multirreferencialidad en lugar de la unidimensionalidad, se le puede concebir como un paradigma posmodernista.

\section{La posmodernidad y el paradigma de la complejidad}

Las relaciones que se pueden encontrar entre el paradigma de la complejidad y la posmodernidad son muchas y muy profundas. Aunque Edgar Morin, fundador del paradigma de la complejidad, no ha aceptado la etiqueta de posmodernista, es muy evidente que sus ideas se pueden interpretar como posmodernistas. Ante esto, Chirinos (2007) afirma que

Morin quien, aunque no se llama a sí mismo postmoderno, retoma temas recurrentes de esta problemática, como es la desfundamentación del conocimiento, el inacabamiento indefinido de la categorización científica y la necesidad de derrumbar las fronteras de las diversas disciplinas científicas, con lo cual los límites 
levantados por el anterior predominio positivista queda prácticamente abolido (p. 372).

Aunque no hay una escuela o tradición claramente definida de lo que se conoce como el posmodernismo y haya autores quienes se desmarcan de esta corriente de pensamiento, es evidente que existe - para ponerlo en términos wittgensteinianos- un aire de familia entre los criterios de algunos autores, entre ellos Morin, por lo que se les puede ubicar dentro de lo que se conoce como el pensamiento posmoderno. A razón de lo anterior, Chirinos (2007), argumenta que:

Morin ha reconocido lo que varios autores postmodernos han repetido insistentemente: el conocimiento no es posible fundamentarlo a la manera clásica, como un sistema filosófico arquitectónicamente construido, a partir de premisas claras, distintas y evidentes, de un modo puramente geométrico o formal. Esto ya no es posible hoy con desarrollos lógicos como el teorema de Gödel, al cual recurren tanto Morin como los postmodernos cuando quieren insistir en la necesidad ilimitada de buscar una demostración a todo término de partida. En otras palabras: tanto el pensamiento complejo de Morin, como todo el pensamiento postmoderno mencionado, han argumentado el inacabamiento indefinido del saber, la imposibilidad del conocimiento total (Lyotard hablaba de la deslegitimación del Espíritu Absoluto hegeliano), la problematicidad de todo enunciado científico (p. 383).

Evidenciadas las relaciones entre el pensamiento complejo y el discurso postmodernista, en el siguiente apartado se va a analizar las referencias a la complejidad que se encuentran en el currículo educativo costarricense. Posteriormente, se va a reflexionar críticamente sobre la inclusión de este modelo en la enseñanza de este país.

\section{El paradigma de la complejidad en la actual Política Educativa de Costa Rica}

En la actual Política Educativa de Costa Rica (MEP, 2017a) se incluyó, por primera vez el paradigma de la complejidad como fundamento de la educación. Explícitamente es poco lo que se menciona en cuanto al significado que tiene este nuevo fundamento para la enseñanza costarricense, 
pero se esperaría que en los distintos planes de las distintas especialidades de enseñanza se especifique más a fondo la manera de cómo se va a concretar ese modelo. Sin embargo, solo en algunos programas como en los de ciencias del MEP (2016) y el MEP (2017b), se pueden encontrar referencias - aunque mínimas - a ese paradigma.

Esta nueva visión de la educación no se ha ido introduciendo de manera repentina ya que han trascurrido décadas en las que poco a poco se han transformado ciertas prácticas educativas motivadas por esta perspectiva. Lo mismo se podría encontrar en otros modelos educativos latinoamericanos en los que, por ejemplo, el paradigma de la complejidad los ha acercado hacia posturas político-educativas que se pueden considerar posmodernas.

América Latina ha tenido una mayor influencia en las ciencias principalmente educativas de este nuevo modelo debido a que desde el año 1999 se creó la "Cátedra Morín" de la UNESCO, con sede en Argentina y en Colombia, la cual ha generado desde entonces estudios e investigaciones sobre este paradigma que se han extendido a otros países como Venezuela, México, Bolivia, Ecuador y Brasil (Velilla, 2002).

No obstante, desde que la educación se concibe como sociocultural, plural, participativa (no dominado por el docente o los libros), crítica, flexible, cambiante, horizontal, heterogénea, no discriminatoria (por sexo, país de procedencia, género, religión, clase social, tradición cultural), según el ritmo y las capacidades de los estudiantes y según el contexto; se podría afirmar que se está ante una educación posmodernista.

Para Aronowitz y Giroux (1991), la posibilidad de opinar e interpretar el aprendizaje por parte del estudiante; la inclusión de distintas voces antes calladas o dominadas como la de los afrodescendientes, las mujeres, las clases marginadas ${ }^{2}$ y la deconstrucción de prácticas educativas tradicionales son muestras de una pedagogía y una educación posmodernista. Sin embargo, los fundamentos del sistema educativo costarricense planteados en la anterior Política Educativa (Consejo Superior de Educación [CSE], 1994), mantenían una visión modernista: humanismo (renacentista), racionalismo (cartesiano) y constructivismo (piagetiano). Con la inclusión del paradigma de la complejidad y el socioconstructivismo como nuevos y

2 Actualmente se podría incluir la de la comunidad LGBTI y las personas inmigrantes. 
principales fundamentos de la enseñanza en la actual Política Educativa (MEP, 2017a), se aprecia una tendencia hacia la visión posmodernista.

\section{Reflexiones críticas}

A continuación, se ofrecen algunas reflexiones críticas como análisis y discusión de lo expuesto anteriormente en cuanto al nuevo paradigma de la educación costarricense:

1. Parece que el paradigma de la complejidad en la actual Política Educativa de Costa Rica solo es un fundamento teórico-conceptual, ya que no refleja en los distintos programas de estudio alguna intención de concebir en algún momento a toda la educación como un sistema, un tejido de constituyentes heterogéneos inseparablemente asociados.

2. Este paradigma propone un conocimiento no fragmentario o mutilado de la realidad, pero la educación costarricense está fragmentada por distintas especialidades, cada una con su propio tiempo, su propio método, sus propios especialistas y su propio programa educativo; aunque existen intenciones de estudiar algunos temas de forma transversal, no existe aún planes para conformar un currículo transdisciplinario que esté más cerca de lo que propone este nuevo paradigma.

3. Después de haber estudiado lo que expone este paradigma se puede decir que no encaja con los otros fundamentos de la Política Educativa, el humanismo y el racionalismo: ambos fundamentos que son concebidos y representativos de la modernidad. La siguiente cita trae a colación una contradicción entre lo que propone el paradigma de la complejidad y lo que se expone como el paradigma del racionalismo, en la actual Política Educativa: "El paradigma del racionalismo, que se sustenta en la razón y en las verdades objetivas como principios para el desarrollo del conocimiento válido, ha sido fundamental en la conceptualización de las políticas educativas costarricenses" (MEP, 2017a, p.10). No hay una verdad única, se desvirtúa el conocimiento meramente objetivo, hay una interdependencia entre el sujeto y el objeto, el conocimiento humano es incompleto, la irracionalidad es importante de valorar, etc. 
4. Asimismo, en esta política el humanismo se concibe de manera renacentista como el humano centro y medida de todo: "La educación, en consecuencia, está centrada en la persona, de manera que sea ella misma evaluadora y guía de su propia experiencia, a través del significado que adquiere su proceso de aprendizaje" (MEP, 2017a, p. 9). Esto último muestra un antropocentrismo que riñe con la visión de la complejidad que presenta Morin (1994) en cuanto al ser humano como una parte de un ecosistema físico y biológico, no solo antropológico, también con las ideas del posthumanismo en cuanto a la superación del antropocentrismo eurocentrista del renacimiento, la respuesta ante el antihumanismo de la Segunda Guerra Mundial, la necesidad de pensar una nueva subjetividad frente a los híbridos (humanos y tecnología), entre otras (Braidotti, 2015). Además, deja de lado las discusiones actuales, que escapan a la visión de la modernidad, sobre el papel actual del ser humano frente a la naturaleza o las discusiones actuales de ética animal en la que los animales no humanos son relevantes en temas emocionales, morales, jurídicos. Es decir, el antropocentrismo de la actual Política Educativa no está en concordancia con nuevas tendencias de pensamiento o temas que actualmente no conciben al ser humano como el centro de discusión o medida de todas las cosas.

5. Este paradigma defiende la flexibilidad para la autocrítica, a la vez que critica las verdades de la ciencia y a los modelos educativos como los eurocentristas ( inclusive cuando Morin es un autor francés), pero ¿soportaría una crítica suficientemente argumentada? Si es así, ¿qué podría esperarse de este hecho?, ¿se debería aceptar como parte de la noción de diálogo, consenso, posibilidad de varias verdades o paradojas? Si se acepta, ¿no quedaría en evidencia un relativismo epistemológico que recuerda al todo vale feyerabendiano, pero con posibles implicaciones negativas como las de un anarquismo en la enseñanza?

6. A pesar de lo anterior, el pensamiento complejo y el pensamiento posmoderno, abren la posibilidad de una mayor apertura de conciencia, esto permite pensar con mayor libertad y criticidad la realidad, con lo cual el futuro se puede visualizar más sustentable y resistente ante determinaciones científicas, filosóficas y políticas tradicionales sobre la educación, la forma de hacer ciencia, el manejo del ambiente, el respeto a la diversidad, entre otros temas. 
7. Dentro de la postmodernidad en la que se vive, el pensamiento complejo representa el paradigma de las ciencias a partir del cual se pretende cambiar o actualizar las distintas teorías previas de este periodo histórico. En el apartado anterior, eso se demostró debido al cambio de fundamentos. Se pasó de fundamentar la educación costarricense del constructivismo piagetiano al paradigma de la complejidad y el constructivismo social. Esto, aunque no se materialice en la Política educativa, MEP (2017a), responde a la influencia que está dejando los enfoques sistémicos e integradores en la educación. García (2000) es un ejemplo de este paso, quien partir de su teoría de sistemas complejos, ha actualizado los planteamientos de Jean Piaget. Para este autor es necesario abordar la teoría epistemológica piagetiana desde un enfoque sistémico que permita reordenar sus postulados hacia la integralidad y la interdisciplinariedad.

8. En cuanto a la posmodernidad en la educación propiamente, algunos autores se mantienen reacios a este discurso, mientras que otros afirman que es más bien favorable y ventajoso. Por ejemplo, Mèlich (2012) afirma que en una educación posmoderna los lenguajes para comunicarse serían diversos, lo cual exacerbaría la ambigüedad. Además, al abandonar las esencias trascendentales quedarían acuerdos contextuales y locales que van a significar el abandono de ciertos aspectos como, primero, los principios absolutos (la noción de verdad única, declaraciones con pretensión de universalidad como la de los derechos humanos, etc.); segundo, los mecanismos sociales de control de discursos en los centros de estudio (las órdenes, evaluaciones y castigos, serían cuestionables desde la subjetividad de cada estudiante); tercero, la objetividad de las distintas disciplinas del saber se podrían perder al restarle exactitud, especialización e imparcialidad a las investigaciones; cuarto, la universalidad que puede tener la ética sería cuestionable y relativa a los contextos locales; entre otros. Sin embargo, para autores como Rojas (2003), la posmodernidad está en contra de los discursos elitistas, totalizantes y dominantes; privilegia la diversidad, la localidad, las nuevas formas de pluralidad de políticas sobre el género, la raza, las clases marginales y la nueva moral sexual. Esto, según Rojas (año de publicación), promueve la deconstrucción de la educación para así crear nuevas y propias prácticas educativas. 
En torno a este último punto, en el contexto de Costa Rica y su Política Educativa, la idea de una educación novedosa y propia a raíz de la posmodernidad no encaja con la insistente adopción de modelos educativos eurocéntricos que impiden la producción de una epistemología y una práctica educativa desde Costa Rica y no desde el extranjero. Lo que plantea Rojas (2003), evidencia la siguiente paradoja: La deconstrucción (que se enmarca en la posmodernidad) promueve la creación de nuevas y propias prácticas educativas en Costa Rica, pero la deconstrucción no es una práctica nueva ni propia de este país. Para crear novedosas y propias formas de enseñanza (o nuevas formas de concebir el conocimiento) no se necesitan métodos o modelos ya existentes de otros contextos, sino métodos o modelos nuevos y propios de cada lugar, en este caso, de Costa Rica, de la región centroamericana $\mathrm{o}$, al menos, de Latinoamérica.

\section{Conclusión}

Es difícil pensar que actualmente alguna teoría, ciencia o investigación no esté permeada, en menor o mayor grado, de lo que se conoce como el posmodernismo o la posmodernidad. Aunque siguen estando vigentes muchas características de la modernidad y de la ciencia moderna, es incontrovertible que esta época o nueva forma de pensamiento está presente en campos como el de las ciencias (la educación), la filosofía, la teología, la pedagogía y otros.

Durante el desarrollo de este ensayo se pudo evidenciar, una vez más, que el sistema educativo costarricense no ha superado el subdesarrollo (intelectual) ni la colonización (del pensamiento), ya que sigue adoptando modelos (educativos) que no son autóctonos y ni siquiera de su región. Según Aronowitz y Giroux (1991), el posmodernismo se propone rechazar el eurocentrismo, el cual busca hablar por toda la humanidad y plantear ideas epistemológicamente fundacionalistas; sin embargo, en Costa Rica se adoptan paradigmas eurocéntricos como los planteados en la Política Educativa, con intenciones fundacionalistas en la educación, lo cual cuál evidencia una contradicción con respecto a la adopción de estos modelos europeos con la intención de erigirse como paradigmas educativos. Pero parece ser que en la posmodernidad las contradicciones son válidas en vista de que no hay una única verdad sino "cientos de verdades relativas que los hombres se reparten” (Mèlich, 2012, p. 35). 
El análisis que puede realizarse de las experiencias de otros modelos educativos formulados fundamentalmente desde este paradigma representaría una investigación comparativa necesaria, que puede brindar argumentos en favor o en contra de la admisión de este tipo de visión en la educación nacional, empero, puede ser pronto (en Costa Rica el paradigma de la complejidad se introdujo en 2017) para investigar sobre la influencia que esta nueva forma de ver la realidad está provocando en la educación del país.

Determinar si la complejidad, como paradigma posmodernista, es apropiada u oportuna ahora o a futuro como fundamento en el campo de la educación en Costa Rica es algo que escapa a los objetivos de este ensayo. Por su parte, como se pudo observar hay opiniones contrarias y mucho en qué reflexionar en relación con este hecho.

Finalmente, como se mencionó en la introducción de este escrito, más que tomar partida a favor o en contra, lo que se pretendió aquí fue exponer de forma clara y crítica - que como se presenta en la Política Educativa de Costa Rica- lo que es el paradigma de la complejidad, su actual relación con la educación de Costa Rica y la corriente de pensamiento o época a la que se le puede adscribir: la posmodernista. 


\section{Referencias}

Aronowitz, S. \& Giroux, H. (1991). Postmodern Education: Politics, Culture, and Social Criticism. University of Minnesota Press.

Braidotti, R. (2015). Lo Posthumano. Gedisa.

Caro A. (2002). El paradigma de la complejidad como salida de la crisis de la posmodernidad. Revista Discurso, 16(17) 69-83. https://bit.ly/2SzOdOu

Chirinos, S. (2007). Complejidad y posmodernidad. Revista Mañongo, 15(29) 371-384. https://bit.ly/34nSyqa

Díaz, E. (2000). Posmodernidad. Biblos.

Gallegos, M. (2016). Una cartografía de las ideas de la complejidad en América Latina: la difusión de Edgar Morin. Latinoamérica, 63, 93128. https://bit.ly/3yLjPko

García, R. (2000). El conocimiento en construcción. De las formulaciones de Jean Piaget a la teoría de sistemas complejos. Gedisa.

Habermas, J. (1985). La modernidad, un proyecto incompleto. En Foster, H. et al. (1985) La posmodernidad. Kairós.

Norris, C. (2008). Postmodernismo. En Honderich, T. (Ed.), Enciclopedia Oxford de Filosofía (p. 932). Tecnos.

Mèlich, J. (2012). Filosofía y educación en la postmodernidad. En Hoyos, G. (Ed.). Filosofía de la educación. Trotta.

Consejo Superior de Educación. (1994). Política Educativa hacia el Siglo $X X I$. https://bit.ly/2Thrc2w

Ministerio de Educación Pública. (2016). Programas de estudio de ciencias. Primero y segundo ciclo de la educación general básica. https://bit.ly/2RQYViU 
Ministerio de Educación Pública. (2017a). Política Educativa. La persona: centro del proceso educativo y sujeto transformador de la sociedad. https://bit.ly/3uBNARq

Ministerio de Educación Pública. (2017b). Programas de estudio de ciencias tercer ciclo de la educación general básica. https://bit.ly/3vBnq2k

Morin, E. (1994). Introducción al pensamiento complejo. https://bit. ly/3i2hwTY

Morin, E. (1999). Los siete saberes necesarios para la educación del futuro. https://bit.ly/34pe51J

Munné, F. (2001). El declive del postmodernismo y el porvenir de la psicología. Revista Cinta moebio, (10), 21-33. https://bit.ly/3yLeae1

Rojas, C. (2003). La filosofía en el debate posmoderno. EUNA.

Solana, J. (2008). El pensamiento complejo como alternativa al neopositivismo y al posmodernismo en antropología. Revista Synergies Monde, 4, 235-243. https://bit.ly/2SBYIjl

Velilla, M. A. (2002). Manual de iniciación pedagógica al Pensamiento complejo. París: ICFES/UNESCO. Recuperado de: http://files.doctorado-en-educacion-2-cohorte.webnode.es/200000055-a98dcaa881/ Manuallniciacion_pedagogica_PC.pdf 